Article

\title{
Blood Metabolite Signature of Metabolic Syndrome Implicates Alterations in Amino Acid Metabolism: Findings from the Baltimore Longitudinal Study of Aging (BLSA) and the Tsuruoka Metabolomics Cohort Study (TMCS)
}

\author{
Jackson A. Roberts ${ }^{1}{ }^{\circledR}$, Vijay R. Varma ${ }^{1}$, Chiung-Wei Huang ${ }^{2}$, Yang An ${ }^{2}$, Anup Oommen ${ }^{3}{ }^{(D}$, \\ Toshiko Tanaka ${ }^{4}\left(\mathbb{D}\right.$, Luigi Ferrucci ${ }^{4}$, Palchamy Elango ${ }^{4}$, Toru Takebayashi ${ }^{5}$, Sei Harada ${ }^{5}(\mathbb{D}$, \\ Miho Iida ${ }^{5}(\mathbb{D})$ and Madhav Thambisetty ${ }^{1, *}$ \\ 1 Clinical and Translational Neuroscience Section, Laboratory of Behavioral Neuroscience, National Institute \\ on Aging, National Institutes of Health, Baltimore, MD 21224, USA; robertsjaa@nih.gov (J.A.R.); \\ vijay.varma@nih.gov (V.R.V.) \\ 2 Brain Aging and Behavior Section, Laboratory of Behavioral Neuroscience, National Institute on Aging, \\ National Institutes of Health, Baltimore, MD 21224, USA; chiung-wei.huang@nih.gov (C.-W.H.); \\ anya@grc.nia.nih.gov (Y.A.) \\ 3 Glycoscience Group, National Centre for Biomedical Engineering Science, National University of Ireland \\ Galway, Galway H91-TK33, Ireland; anup.mammen@gmail.com \\ 4 Translational Gerontology Branch, National Institute on Aging, NIH, Baltimore, MD 21224, USA; \\ tanakato@mail.nih.gov (T.T.); ferruccilu@grc.nia.nih.gov (L.F.); elangop@mail.nih.gov (P.E.) \\ 5 Department of Preventive Medicine and Public Health, Keio University, Tokyo 160-8282, Japan; \\ ttakebayashi@a3.keio.jp (T.T.); seiharada@keio.jp (S.H.); mihoiida1029@gmail.com (M.I.) \\ * Correspondence: thambisettym@mail.nih.gov; Tel.: +1-(410)-558-8572; Fax: +1-(410)-558-8302
}

Received: 19 December 2019; Accepted: 10 February 2020; Published: 13 February 2020

\begin{abstract}
Rapid lifestyle and dietary changes have contributed to a rise in the global prevalence of metabolic syndrome (MetS), which presents a potential healthcare crisis, owing to its association with an increased burden of multiple cardiovascular and neurological diseases. Prior work has identified the role that genetic, lifestyle, and environmental factors can play in the prevalence of MetS. Metabolomics is an important tool to study alterations in biochemical pathways intrinsic to the pathophysiology of MetS. We undertook a metabolomic study of MetS in serum samples from two ethnically distinct, well-characterized cohorts—-the Baltimore Longitudinal Study of Aging (BLSA) from the U.S. and the Tsuruoka Metabolomics Cohort Study (TMCS) from Japan. We used multivariate logistic regression to identify metabolites that were associated with MetS in both cohorts. Among the top 25 most significant (lowest $p$-value) metabolite associations with MetS in each cohort, we identified 18 metabolites that were shared between TMCS and BLSA, the majority of which were classified as amino acids. These associations implicate multiple biochemical pathways in MetS, including branched-chain amino acid metabolism, glutathione production, aromatic amino acid metabolism, gluconeogenesis, and the tricarboxylic acid cycle. Our results suggest that fundamental alterations in amino acid metabolism may be central features of MetS.
\end{abstract}

Keywords: metabolic syndrome; metabolomics; amino acids; fasting glucose; triglycerides; waist circumference; blood pressure 


\section{Introduction}

Metabolic syndrome (MetS) is a cluster of risk factors that raises an individual's risk for heart disease, diabetes, stroke, and chronic neurodegenerative disease [1-4]. MetS is defined as having 3 or more of 5 individual risk factors including elevated waist circumference, fasting serum/plasma glucose concentration, triglyceride levels, and blood pressure and reduced serum/plasma high-density lipoprotein (HDL) cholesterol [5]. In the United States, more than one-third of adults and more than one-half of those 60 years or older have MetS [6]. Additionally, the global spread of the Westernized lifestyle has increased the global burden of metabolic diseases, representing a substantial public health concern as more societies become sedentary and transition to more unhealthy diets [7,8]. In Japan, for instance, government estimates suggest that nearly one-third of males and one-tenth of females 20 years or older have MetS and expectations suggest that the prevalence will likely increase in the coming years [9]. Presence of MetS further inflates the per-patient cost of treatment for those with hypertension, and overall patient-specific health costs increase with the accumulation of each additional MetS risk factor [10].

Part of the health and cost burden imposed by MetS stems from its association with increased morbidity and mortality of other diseases. MetS has been shown to double the risk of fatal cardiovascular events [11]; has been shown to increase the risk of cardiovascular disease and type II diabetes mellitus (T2DM) [1,2]; and is associated with adverse neurological outcomes, including increased risk of cognitive decline, vascular dementia, and Alzheimer's disease (AD) [12-14]. MetS likely increases risk of these conditions through chronic inflammation, hyperinsulinemia, dyslipidemia, and oxidative stress [15]. Identifying the biologic pathways and mechanisms underlying MetS is essential to developing biomarkers and interventions for multiple chronic and neurological diseases.

Despite prior work, the pathophysiology of MetS remains unclear. Recently, high-throughput omics has emerged as an important tool for understanding disease-specific cellular processes [16]. Metabolomics of blood in particular may improve the mechanistic understanding of disease pathology [17]. Metabolite profiling of MetS can help characterize altered metabolic processes, which may lead to earlier diagnosis and intervention as well as improved personalized treatments [18].

Prior work has identified blood serum metabolites that characterize early phenotypes of MetS prior to diagnosis [19]. Metabolomics has also been used to discriminate between overweight and obese individuals with and without MetS [20,21], suggesting some diagnostic utility. Other studies established blood metabolite profiles of different dietary patterns in patients with MetS [22,23], indicating that the approach may be useful for studying the impact of lifestyle on disease processes. Metabolomics studies in blood tissue have additionally identified multiple biochemical pathways in MetS, most notably altered amino acid metabolism [24].

Cross-cultural and multiethnic studies may yield significant insight into biochemical processes associated with MetS that are fundamental to the disease etiology. These studies may provide important information regarding the interaction between genes and environment in the development of disease and may shed light on fundamental biologic features of disease pathology. Prior cross-cultural studies have examined clustering of MetS risk factors by ethnicity [25] as well as shared and distinct metabolite associations with type II diabetes mellitus (T2DM) [26,27]. However, a metabolomic approach in two ethnically distinct longitudinal cohorts has not yet been applied to identify a metabolite signature of MetS.

The purpose of the present study is to comprehensively explore a serum metabolite signature of MetS in two ethnically distinct cohorts of older individuals-the Baltimore Longitudinal Study of Aging (BLSA) from the United States and Tsuruoka Metabolomics Cohort Study (TMCS) from Japan. These distinct cohorts allow for cross comparisons that can help identify metabolic alterations that may be fundamental to MetS. Specifically, we utilized capillary electrophoresis time-of-flight mass spectrometry (CE-TOFMS) to assay charged and hydrophilic blood serum metabolites and examined their associations with MetS. To our knowledge, our study is one of the first to utilize this omics platform in a large, multiethnic study of MetS. 


\section{Results}

\subsection{Participants}

Table 1 summarizes the demographic characteristics of participants from the BLSA and TMCS cohorts. Comparing the overall samples between the two cohorts, as shown in columns 2 and 3 of Table 1, BLSA participants were, on average, older and more likely to have never smoked. The BLSA participants were more likely male compared to TMCS. The prevalence of MetS was similar between groups, but BLSA participants had a greater prevalence of elevated triglycerides and reduced HDL-C. Overall, TMCS participants had a greater prevalence of elevated waist circumference, elevated fasting glucose, and elevated blood pressure. Considering the continuous values for risk factors, on average, the BLSA cohort had a larger waist circumference and lower HDL cholesterol than the TMCS cohort. The TMCS cohort, on average, had higher fasting glucose and higher systolic and diastolic blood pressures. Average triglyceride levels did not differ between the two cohorts. Regarding prescription drug use, TMCS participants were more likely to use antihypertensive medications, while BLSA participants were more likely to use lipid-lowering medications. Both cohorts were similar in terms of use of diabetes medication.

Table 1. Demographic characteristics of the Baltimore Longitudinal Study of Aging (BLSA) and Tsuruoka Metabolomics Cohort Study (TMCS) cohorts for overall samples and only participants with metabolic syndrome (MetS): Two sample t-tests were used for comparison of continuous variables. Chi-square tests were utilized for comparison of categorical variables. * Significant difference between cohorts at $p<0.05$ in the full sample and + comparisons were significant at $p<0.05$ in samples restricted to participants with MetS. Physical activity questionnaires were not directly comparable, and thus, between group differences were not calculated.

\begin{tabular}{|c|c|c|c|c|}
\hline & $\begin{array}{c}\text { BLSA (Total } \\
\text { Sample) }(n=252)\end{array}$ & $\begin{array}{c}\text { TMCS (Total } \\
\text { Sample) }(n=644)\end{array}$ & $\begin{array}{l}\text { BLSA MetS } \\
\quad(n=106)\end{array}$ & $\begin{array}{c}\text { TMCS MetS } \\
(n=274)\end{array}$ \\
\hline Age, Mean (SD) & $73.8(8.8) *$ & $69.4(2.2) *$ & $72.6(7.9)^{\dagger}$ & $69.4(2.3)^{\dagger}$ \\
\hline Female, n (\%) & $120(47.2) *$ & $359(55.8) *$ & $46(43.4)^{\dagger}$ & $190(69.3)^{\dagger}$ \\
\hline White, n (\%) & $210(83.3)$ & - & $82(77.4)$ & - \\
\hline Never smoke, n (\%) & $112(44.4) *$ & $424(65.8) *$ & $60(56.6)^{\dagger}$ & $205(74.8)^{\dagger}$ \\
\hline Physical Activity (SD) & $87.3(61.5)$ & $17.5(14.6)$ & $80.6(58.8)$ & $16.8(13.5)$ \\
\hline DASH Score (SD) & - & $2.69(0.7)$ & - & $2.71(0.7)$ \\
\hline Storage time, Mean (SD) & $12.4(9.4)$ & - & $11.1(9.2)$ & - \\
\hline Metabolic syndrome, n (\%) & $106(42.1)$ & $274(42.6)$ & - & - \\
\hline Elevated waist circumference, n (\%) & $83(32.9) *$ & $276(42.9) *$ & $64(60.4)$ & $194(70.8)$ \\
\hline Waist circumference, Mean (SD) & $35.9(4.8) *$ & $32.6(3.2) *$ & $38.4(4.4)^{\dagger}$ & $34.2(3.2)^{\dagger}$ \\
\hline Elevated triglyceride level, n (\%) & $121(48.0) *$ & $241(37.4) *$ & $93(87.7)^{\dagger}$ & $211(77.0)^{\dagger}$ \\
\hline Triglyceride level, Mean (SD) & $104.5(59.6)$ & $105(62.4)$ & $133.7(73.5)$ & $124.6(79.1)$ \\
\hline Hyperlipidemia drug use, n (\%) & $93(37.4) *$ & $187(29.0) *$ & $70(66.0)$ & $175(63.9)$ \\
\hline Reduced HDL cholesterol, n (\%) & $138(54.8) *$ & $209(32.4) *$ & $97(91.5)^{\dagger}$ & $190(69.3)^{\dagger}$ \\
\hline HDL cholesterol, Mean (SD) & $56.1(16.4) *$ & $67.4(18.0) *$ & $49.6(14.6)^{\dagger}$ & $64.3(18.7)^{+}$ \\
\hline Elevated Blood Pressure, n (\%) & $134(53.2) *$ & $453(70.3) *$ & $81(76.4)^{\dagger}$ & $237(86.5)^{\dagger}$ \\
\hline SBP, Mean (SD) & $124.6(20.2) *$ & $132.8(18.4) *$ & $125.3(18.9)^{\dagger}$ & $137.4(18.0)^{\dagger}$ \\
\hline DBP, Mean (SD) & $71.3(12.3) *$ & $75.6(10.7) *$ & $71.8(12.7)^{\dagger}$ & $76.9(10.0)^{\dagger}$ \\
\hline Hypertension drug use, n (\%) & $56(22.5) *$ & $297(46.1) *$ & $42(39.6)^{\dagger}$ & $165(60.2)^{+}$ \\
\hline Elevated fasting glucose, n (\%) & $97(39.0) *$ & $319(49.5) *$ & $64(60.4)$ & $186(67.9)$ \\
\hline Fasting glucose, Mean (SD) & $99.6(16.7) *$ & $103.2(15.9) *$ & $106.5(19.9)$ & $107.1(17.1)$ \\
\hline Diabetes drug use, n (\%) & $18(7.2)$ & $60(9.3)$ & $17(16.0)$ & $38(13.9)$ \\
\hline
\end{tabular}

BLSA: Baltimore Longitudinal Study of Aging; TMCS: Tsuruoka Metabolomics Cohort Study; SBP: systolic blood pressure; DBP: diastolic blood pressure; SD: standard deviation; DASH: Dietary Approaches to Stop Hypertension; HDL: high-density lipoprotein. 
The comparisons between each cohort among individuals with MetS are displayed in columns 4 and 5 of Table 1 and are summarized in Figure 1. As shown in Figure 1, TMCS participants with MetS displayed a greater prevalence of elevated blood pressure, a lower prevalence of elevated triglycerides, and reduced HDL-C relative to BLSA participants with MetS. Similar to the comparison of the overall samples, individuals with MetS in the TMCS cohort displayed a higher use of antihypertensive medication. The use of diabetes medication and lipid-lowering medications was similar between the MetS subgroups of each cohort.

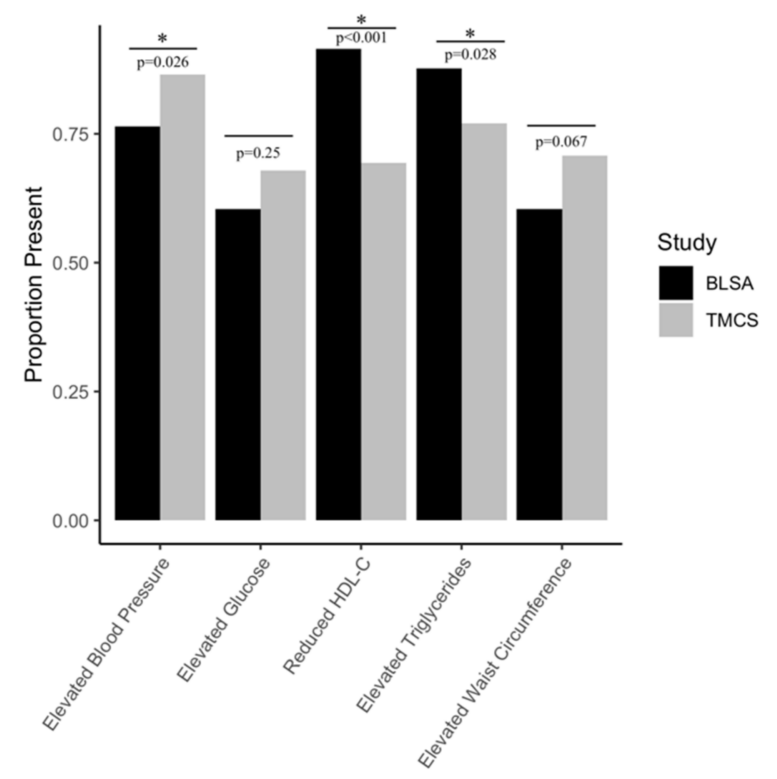

Figure 1. Distribution of individual MetS risk factors among participants with MetS in each cohort:

* Significant difference at $p<0.05$ between risk factor prevalence according to chi-square tests.

\subsection{Metabolite Concentrations and Classifications}

Table S2 includes the median metabolite concentrations for BLSA and TMCS and the false discovery rate (FDR)-adjusted $p$-values indicating the significance of differences between the two cohorts. The majority of metabolites (76/82) was significantly different after FDR correction between the two cohorts.

We classified metabolites according to the Human Metabolome Database (HMDB; http://www. hmdb.ca/) designations (see Table S1): 44 metabolites were classified as carboxylic acids and derivatives; 8 metabolites were classified as fatty acyls; 5 metabolites were classified as organooxygen compounds; 4 metabolites were classified as hydroxy acids and derivatives; 3 metabolites were classified as keto acids and derivatives; and 3 metabolites were classified as organonitrogen compounds. The additional 15 metabolites were each classified into distinct categories with two or fewer metabolites including amines, benzenes and substituted derivatives, glycerophospholipids, imidazopyrimidines, indoles and derivatives, organic sulfonic acids and derivatives, organic sulfuric acids and derivatives, pyridines and derivatives, and pyrimidine nucleosides. Three metabolites were unclassified by HMDB.

\subsection{Associations with Metabolic Risk Factors}

In Table S3, we present the ranked results of all logistic regression models for both TMCS and BLSA cohorts separately. As described in the Methods section, we ranked metabolites based on the likelihood of a type I error (i.e., $p$-value) and identified the top 25 metabolites in each cohort with the lowest likelihood of a type I error (i.e., smallest $p$-value). We indicated with an * if a metabolite in the top 25 for one cohort was also in the top 25 for the other cohort, and we indicated with a t if the metabolite was unique to the top 25 in one cohort. In the BLSA, 17 metabolites were significantly associated with MetS, 16 were significantly associated with elevated waist circumference, 7 were significantly 
associated with elevated fasting glucose, 4 were significantly associated with elevated triglycerides, and no metabolites were significantly associated with reduced HDL-C or elevated blood pressure after FDR correction. In the TMCS cohort, 33 metabolites were significantly associated with MetS, 21 were significantly associated with elevated waist circumference, 21 were significantly associated with elevated triglycerides, 19 were associated with elevated fasting glucose, 6 were associated with reduced HDL-C, and 4 were associated with elevated blood pressure after FDR correction.

In Figure 2, we summarize the results of the logistic regression models measuring the association between metabolites and MetS in both cohorts for the 25 metabolites with the lowest likelihood of a type I error (i.e., smallest $p$-value). Of the 25 most significant metabolites, 18 metabolites were in the top 25 for both BLSA and TMCS cohorts while 7 were unique within each cohort. Results remained consistent in three different sensitivity analyses: (1) adjusting models for smoking status, physical activity, and diet quality (Table S4); (2) sex-stratified analyses (Table S5) in which fewer metabolites remained significant after FDR correction, likely due to reduced statistical power; and (3) excluding imputed values (Table S6).
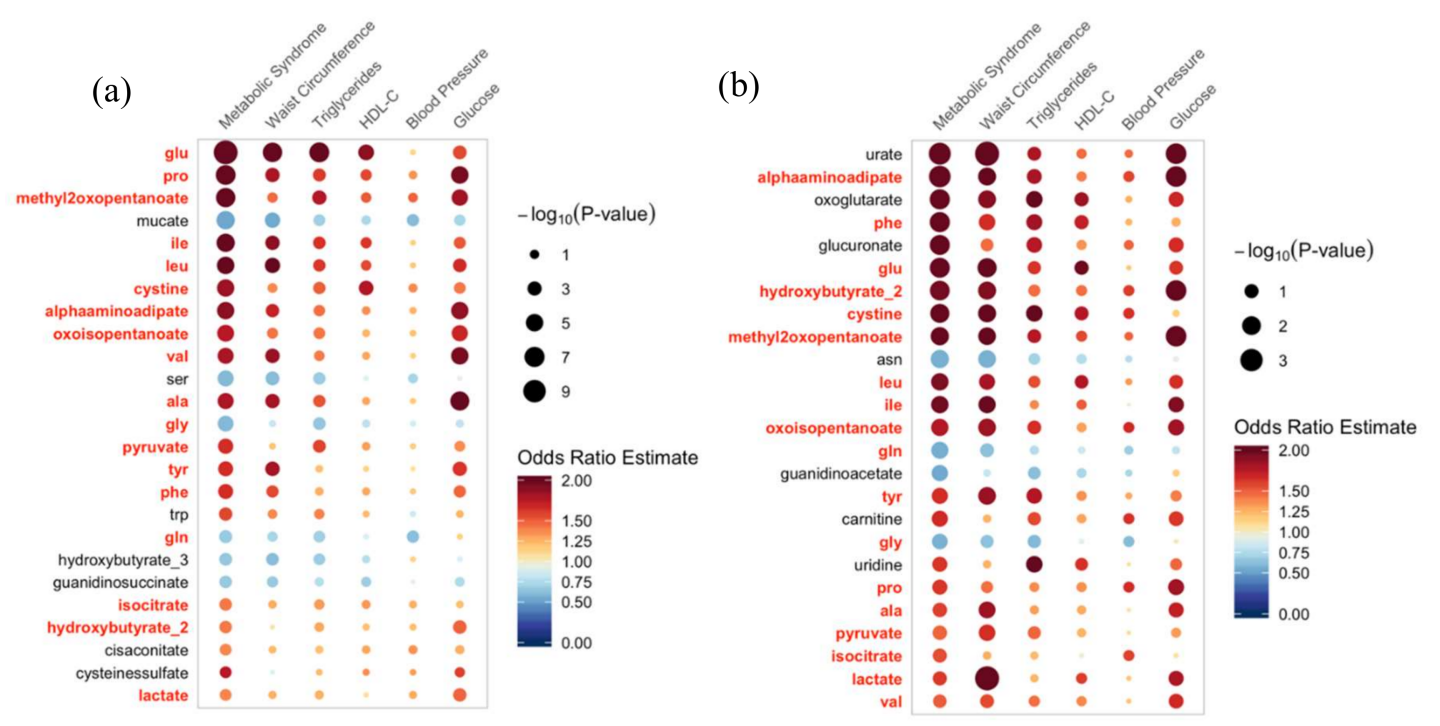

Figure 2. Logistic regression model results for the top 25 metabolites with the lowest likelihood of a type I error (i.e., smallest $p$-value) from the logistic regression model measuring the association between metabolites and MetS in (a) TMCS and (b) BLSA. p-values are false-discovery rate (FDR) corrected for multiple comparisons, and results are adjusted for age and sex in both cohorts as well as for storage time in the BLSA cohort. The $y$-axis indicates the serum metabolite concentration used as the predictor in the model and is ordered (i.e., ranked) from smallest to largest $p$-value within the top 25. The $x$-axis indicates the outcomes of MetS and its individual risk factors. The size of each circle corresponds to the significance of the association, with a larger circle indicating a smaller $p$-value. The color of each circle indicates the value of the odds ratio estimate with blue indicating an OR $<1$ and red indicating an OR $>1$. Red, bolded font indicates the metabolite was among the top 25 in both cohorts. Abbreviations: glu: glutamate; pro: proline; ile: isoleucine; leu: leucine; val: valine; ser: serine; ala: alanine; gly: glycine; tyr: tyrosine; phe: phenylalanine; trp: tryptophan; gln: glutamine; and asn: asparagine; BLSA: Baltimore Longitudinal Study of Aging; TMCS: Tsuruoka Metabolomics Cohort Study.

\subsection{Metabolite Classes Associated with MetS}

A number of metabolite classes were represented among the 25 metabolites most significantly associated with MetS in each cohort. These were classified a priori using HMDB class designations reported above in Section 2.2. Of the 18 metabolites shared in the top 25 between cohorts, 13 represented carboxylic acids and their derivatives. The remaining 5 metabolites were keto acids and their derivatives ( 2 metabolites), hydroxy acids and their derivatives ( 2 metabolites), and pyruvate, 
which was unclassified by HMDB. The 7 metabolites unique to the TMCS cohort in the top 25 were classified as carboxylic acids and their derivatives (4 metabolites), hydroxy acid and their derivatives ( 1 metabolite), indole and their derivatives ( 1 metabolite), and organooxygen compounds ( 1 metabolite). The 7 metabolites unique to the BLSA cohort among the top 25 represented carboxylic acids and their derivatives ( 2 metabolites), pyrimidine nucleosides (1 metabolite), keto acid and their derivatives (1 metabolite), imidazopyrimidine (1 metabolite), organonitrogen compounds (1 metabolite), and organooxygen compounds (1 metabolite).

Of the 18 metabolites overlapping between BLSA and TMCS, we additionally categorized them into metabolic pathways based upon the HMDB pathway database and a literature search of metabolite-associated pathways (see the Methods section for a description of pathway classification relevant to MetS and related conditions). Classification by this methodology resulted in the identification of 7 primary pathways: aromatic amino acid metabolism, amino acid metabolism, lysine degradation, tricarboxylic acid cycle, glutathione production, gluconeogenesis, and branched-chain amino acid metabolism. The class and primary pathways of the 18 metabolites overlapping between BLSA and TMCS in the top 25 are summarized in Table 2.

Table 2. Shared metabolites among the top 25 metabolites with the lowest probability of type I error from the logistic regression models measuring the association between metabolites and MetS: The class of each metabolite was determined by Human Metabolome Database (HMDB) classification, and the primary pathway was determined by the HMDB pathway database tool in addition to a literature search identifying pathways relevant to MetS and related conditions.

\begin{tabular}{ccc}
\hline Metabolite & Class & Primary Pathway * \\
\hline Lactate & Alpha hydroxy acids & Gluconeogenesis \\
2-hydroxybutyrate & Alpha hydroxy acids & Glutathione production \\
Pro & Carboxylic acids & Amino acid metabolism \\
Phe & Carboxylic acids & Aromatic amino acid metabolism \\
Tyr & Carboxylic acids & Aromatic amino acid metabolism \\
Ile & Carboxylic acids & BCAA metabolism \\
Leu & Carboxylic acids & BCAA metabolism \\
Val & Carboxylic acids & BCAA metabolism \\
Ala & Carboxylic acids & Gluconeogenesis \\
Glu & Carboxylic acids & Glutathione metabolism \\
Cystine & Carboxylic acids & Glutathione metabolism \\
Gly & Carboxylic acids & Glutathione metabolism \\
Gln & Carboxylic acids & Glutathione metabolism \\
Alphaaminoapidate & Carboxylic acids & Lysine degradation \\
Isocitrate & Carboxylic acids & Tricarboxylic acid cycle \\
Methyl-2-oxopentanoate & Short-chain keto acids & BCAA metabolism \\
Oxoisopentanoate & Short-chain keto acids & BCAA metabolism \\
Pyruvate & Unclassified & Gluconeogenesis \\
\hline
\end{tabular}

BCAA: branched-chain amino acid; * the likely pathway of primary biologic relevance to MetS and related conditions.

\section{Discussion}

In this study, we compared U.S. and Japanese populations of older individuals in order to identify a serum metabolite signature of MetS that may be intrinsic to the syndrome despite markedly different population-specific characteristics and distribution of individual risk factors. We identified 18 metabolites that were common among the 25 metabolites with the strongest associations with MetS in each cohort; these metabolites were mainly carboxylic acids, hydroxy acids, and keto acids. Overwhelmingly, the shared associations identified in this study represented amino acids-which account for 11 of the 13 carboxylic acids among the 18 shared metabolites-or metabolites implicated in amino acid metabolism. Despite numerous differences between cohorts including metabolite concentrations and prevalence of MetS risk factors, we identified alterations in overlapping metabolites across cohorts, suggesting that fundamental alterations in amino acid metabolism may be intrinsic to 
MetS pathophysiology. This signature suggests a set of metabolites and associated pathways that may be important for improved diagnosis and targeted therapeutic approaches.

Interestingly, while the prevalence of MetS was similar between TMCS and BLSA cohorts, the prevalence of individual risk factors in the overall sample as well as among individuals with MetS varied between cohorts. This finding is consistent with prior studies that indicate a cross-cultural heterogeneity in the combination of the risk factors in MetS [25]. Part of these differences in risk factor prevalence may stem from differences in drug prescription guidelines and practice between the U.S. and Japan, since we reported differences in drug usage prevalence between cohorts. It has previously been reported that cholesterol-lowering statins are underutilized in Japanese patients at risk for cardiovascular disease, and differences in antihypertensive medication prescription have been reported, suggesting that drug prescription guidelines and practices could underly this difference [28-30]. It is important to note that we do not report pretreatment values of these measures and are therefore unable to identify with certainty how differences in drug usage may impact risk factor differences. Finally, contrary to prior research indicating a higher smoking prevalence in Asian countries [31], we report higher rates of smoking among U.S. participants in this study.

Our findings suggest that, despite the heterogeneity in the prevalence of MetS risk factors across both cohorts and differences in serum concentrations in over $92 \%$ of the metabolites measured in this study, the majority of each cohort's most significant metabolite associations with MetS was similar. The 18 common and overlapping metabolites were either amino acids or metabolites directly or indirectly related to amino acid metabolism, suggesting that amino acid metabolism and associated pathways may be fundamental to the biologic processes that may underlie MetS. In particular, the amino acid metabolic pathways represented in these results potentially implicate branched-chain amino acid (BCAA) metabolism and glutathione (GSH) synthesis in the pathogenesis of MetS.

Circulating concentrations of both serum and plasma BCAAs, which include valine, isoleucine, and leucine, have been previously linked to obesity and insulin resistance [32-34]. In a Chinese cohort, BCAAs were associated with overall MetS as well as elevated fasting glucose, elevated triglycerides, and reduced HDL-C [35]. When BCAA metabolism becomes dysregulated, as in MetS, the breakdown products of valine, isoleucine, and leucine may accumulate and exert negative metabolic effects. This is evidenced by the previous finding that a genetic disposition to impaired BCAA metabolism confers an increased risk for type II diabetes mellitus [36]. Notably, branched-chain alpha-ketoacid (BCKA; direct catabolic products of the BCAAs) administration has been demonstrated to induce mitochondrial dysfunction in diverse tissues [37-39]. In our study, all three BCAAs were positively associated with MetS in both cohorts and the BCKAs methyl-2-oxopentanoate and oxoisopentanoate were also positively associated with MetS. This suggests that BCAA metabolism may be fundamentally implicated in MetS and confirms that BCAAs and BCKAs may represent markers of the disease in diverse populations. Interestingly, both BCAAs and BCKAs have been associated with MetS in a previous TMCS cohort of postmenopausal women [40].

More generally, BCAAs have been frequently studied in the context of metabolic disorders and obesity. Among healthy-weight individuals, BCAAs appear to exert beneficial effects, including a decreased risk of obesity, increased muscle mass, potential improvements in glucose sensitivity, and possible therapeutic effects for patients with hepatic cirrhosis and encephalopathy [41-44]. However, long-term exposure to elevated BCAAs stimulates hyperphagia and obesity, has been correlated positively with LDL and triglyceride levels and negatively with HDL-C, and directly inhibits the TCA cycle through BCKA accumulation $[43,45,46]$. In prior in vitro studies using rat cortical slices, BCKA accumulation has also been demonstrated to reduce neuronal glutamate uptake [47], which has been identified as a potential pathological feature of Alzheimer's disease (AD) [48]. In addition, a genetic predisposition to elevated isoleucine has been found to increase risk of AD, and leucine has previously been shown to promote tau accumulation via a mammalian target of rapamycin (mTOR)-dependent mechanism [49,50]. An altered BCAA metabolism could therefore represent a potential mechanistic link between MetS and AD [51]. 
Oxidative stress likely plays a key role in the pathology of both AD and obesity-related disorders [52-54] among older adult populations like those included in this study. One essential mechanism for combatting oxidative stress is the production of GSH, a molecule with numerous antioxidant roles for which homeostasis is disrupted in both AD and obesity $[55,56]$. Interestingly, our recent work using the CE-MS platform to measure brain tissue metabolite concentrations in the BLSA autopsy study identified a positive association between GSH and cystine, a GSH precursor, in AD, suggesting a disease-specific, chronic inflammatory state [57]. In the present study, several amino acids essential to GSH synthesis were associated with MetS. Most notably, cystine and glutamate displayed positive associations with MetS while glycine displayed a negative association. While cystine is considered a rate-limiting metabolite for the synthesis of GSH, elevated extracellular glutamate inhibits the uptake of cystine and reduces GSH synthesis [58]. Independently, cystine has been associated with obesity and insulin resistance [59]. Additionally, 2-hydroxybutyrate was positively associated with MetS in this study and plays an important role in the GSH synthetic pathway. Specifically, 2-hydroxybutyrate is a byproduct of cystine formation, and its association with MetS may represent an increased flux through GSH synthesis in response to oxidative stress [60]. When its levels are reduced, glycine may become the limiting factor in GSH production as suggested by the finding that glycine supplementation restores GSH levels and reduces oxidative stress [61]. Serine, which was associated with a decreased risk of MetS in the TMCS cohort only, has been shown to play a role in GSH synthesis through its conversion to glycine [62]. Another amino acid, glutamine, that was associated with a decreased risk of MetS in both cohorts has additionally been shown to drive production of glutathione [63]. As a whole, our results suggest that MetS implicates pathways associated with chronic oxidative stress owing to the positive associations of several metabolites from the GSH synthesis pathway with MetS. Furthermore, glycine availability may be a limiting factor in GSH production under these conditions, since glycine demonstrated a negative association with MetS.

Our results further implicate the importance of gluconeogenesis and particularly the glucose-alanine cycle in MetS. In this study, alanine, pyruvate, and lactate all displayed positive associations with MetS across cohorts. Alanine is the predominant glucogenic amino acid and is typically released from muscle under conditions in which glucose is scarce. In peripheral tissues, alanine is produced through transamination of pyruvate and is then converted back to pyruvate and glucose via gluconeogenesis in the liver [64]. Lactate is typically produced from pyruvate under conditions of anaerobic glycolysis and is similarly reconverted to pyruvate for gluconeogenesis in the liver. Subcutaneous fat has been demonstrated to be a source of excess lactate in obese individuals, suggesting altered metabolism in the obese [65]. The positive associations of alanine, pyruvate, and lactate with MetS suggests that alterations in glycolytic flux are likely central features of MetS.

Interestingly, it has been shown that disruption of pyruvate entry into the tricarboxylic acid (TCA) cycle causes an increased rate of conversion of glutamine-which was identified as protective against MetS in this study-into oxoglutarate, a TCA cycle intermediate [66]. Increased utilization of amino acids for the TCA cycle under pathological conditions could reduce their availability for other key biochemical pathways, such as GSH synthesis discussed previously. More general alterations in the TCA cycle may be fundamental to MetS, as we identified that isocitrate was positively associated with the disease in both cohorts. This is not surprising, as increased anaplerotic flux generating TCA intermediates is a hallmark feature of obesity and insulin resistance [67].

We additionally identified an alteration in aromatic amino acid metabolism, indicated by the positive association of phenylalanine and tyrosine with MetS across cohorts. Tyrosine is produced through hydroxylation of phenylalanine, and both metabolites have previously been associated with obesity [68]. These amino acids may become elevated as a result of competition with BCAAs for the same large neutral amino acid transporter or inhibition of tyrosine aminotransferase by cystine $[32,69]$. Interestingly, phenylalanine and tyrosine function as precursors for catecholamines in the brain, presenting a potential link between the metabolic dysfunction of MetS and common neurological diseases $[70,71]$. 
Lastly, we identified that alpha-aminoadipate, a breakdown product of lysine degradation, was associated with MetS in both cohorts. This metabolite has previously been identified as a marker distinguishing between metabolically healthy and unhealthy obese individuals and has been considered a biomarker of diabetes [72,73].

In a prior study, we utilized metabolomics to identify classes of metabolites associated with MetS in a study including similar cohorts from the BLSA and TMCS [74]. In that study, we performed flow injection analysis-tandem mass spectrometry and liquid chromatography-tandem mass spectrometry to quantify 167 metabolites spanning a wide variety of polar and hydrophobic compounds. We identified that phosphatidylcholines-acyl-alkyls, sphingomyelins, and hexoses were associated with MetS in both cohorts, suggesting that these classes of metabolites may represent fundamental markers of the syndrome. Furthermore, we measured 27 of the 82 metabolites quantified in this study, some of which were associated with MetS in this study but not our prior work. There may be a few explanations for this disparity between our results. First, the present study makes use of much larger sample sizes for both cohorts, nearly six times larger for TMCS and twice as large for BLSA. This increased the statistical power of our analyses and thus improved the possibility that additional metabolite associations were captured. In addition, the current study utilizes a different metabolomics platform than our previous work, quantifying metabolites by CE-TOFMS as opposed to LC-MS, which may explain some variation in our results.

Our study has several limitations. First, the TMCS cohort represented a narrower age range than the BLSA cohort and were from the same city and prefecture in Japan, suggesting that the BLSA cohort was more heterogenous with respect to a number of unmeasured environmental and ethnicity-related factors. Additionally, the TMCS cohort is likely more representative of the general Japanese population than the BLSA cohort is of the U.S. population, since the BLSA participants are predominantly Caucasian, well educated, and relatively healthy. In addition, the metabolomics platform we utilized only allowed us to measure a subset of the serum metabolome. Furthermore, while our sample provided sufficient power to detect significant associations between metabolites and MetS, a larger sample size may have allowed for the detection of other novel metabolites that we did not detect in our current analyses. Lastly, the cross-sectional nature of the present study prevents us from making inferences regarding metabolic predictors of MetS onset or how longitudinal changes in metabolite concentrations may reflect disease processes.

In conclusion, our results highlight the importance of several altered amino acid metabolic pathways in MetS. Specifically, we confirm through analysis in two ethnically distinct cohorts that BCAA metabolism and GSH synthesis may represent central pathways contributing to MetS and additionally note alterations in gluconeogenesis, TCA cycle, aromatic amino acid metabolism, and lysine degradation. It is possible that these alterations in amino acid metabolism may be the result of mitochondrial dysfunction coupled with protein breakdown, which have previously been discussed as causal mechanisms in amino acid alterations [68,75]. These alterations, however, take place across disparate biochemical pathways, suggesting that MetS likely represents a state of chronic, systemic metabolic dysregulation. The findings of this study are relevant for future studies examining altered metabolic flux through these pathways in MetS as well as an understanding of shared pathways across MetS and other diseases associated with aging.

\section{Methods}

\subsection{Participants}

The National Institute on Aging's (NIA) BLSA is one of the longest running scientific studies of human aging in the U.S. [76]. This observational study began in 1958 and includes longitudinal clinical, radiological, and laboratory evaluations on community-dwelling volunteer participants. The individuals in this study were participants in the neuroimaging sub-study of the BLSA [77]. Written informed consent was obtained at each visit for all BLSA participants. The BLSA study protocol 
has ongoing approval from the Institutional Review Board of the National Institute of Environmental Health Science, National Institutes of Health ("Early Markers of Alzheimer's Disease (BLSA)", IRB No. 2009-074).

The Tsuruoka Metabolomics Cohort Study (TMCS) is a population-based study of residents from Tsuruoka City, Yamagata Prefecture, Japan that began in 2012. Participants were recruited from annual municipal or worksite health checkup programs in the city. The individuals in this study were a subset from the baseline survey participants of the TMCS [78]. Written informed consent was obtained from all TMCS participants. The TMCS study protocol has ongoing approval from the Medical Ethics Committee of the School of Medicine, Keio University, Tokyo, Japan (Approval No. 20110264; original approval date: 6 December 2011; latest update: 2 December 2019).

\subsection{Blood Samples}

In BLSA participants, blood samples were collected at the NIA Clinical Research Unit in Harbor Hospital, Baltimore, MD. Collection and processing details have been described previously [79]. Briefly, venous blood samples were collected (after an overnight fast) between 6 and 7 AM. Serum samples were aliquoted into $0.5-\mathrm{mL}$ volumes in Nunc cryogenic tubes (Rochester, NY, USA) and stored at $-80{ }^{\circ} \mathrm{C}$. Samples were not subject to any freeze-thaw cycles prior to metabolomic assays. The average storage time of BLSA serum samples prior to thaw for quantitative metabolomics was 12.39 years (SD: 9.41). The sample included 252 participants.

For TMCS participants, blood serum samples were collected during annual health checkups. Details on collection and processing have been published previously $[40,78]$. Briefly, venous blood samples were collected (after an overnight fast) between 8:30 and 10:30 AM. Serum samples were collected with serum-separating medium and assayed immediately. Storage time was less than $6 \mathrm{~h}$ and did not vary across TMCS participants. The sample included 644 participants.

\subsection{Metabolites}

Non-targeted metabolomics was carried out in this study using capillary electrophoresis time-of-flight mass spectrometry (CE-TOFMS) for quantification of metabolites. CE-TOFMS captures a broad range of charged and hydrophilic metabolites, including amino acids, organic acids, ketoacids, and a number of other metabolite classes which represent a variety of biological pathways. Compared to other methods, CE-TOFMS requires a small sample size with robust sensitivity and high resolution of results [80].

\subsubsection{Metabolite Extraction}

As described previously [81], metabolite extraction was completed at Keio University, Tokyo, Japan. Briefly, samples were thawed and $100-\mu \mathrm{L}$ serum aliquots were placed in $900-\mu \mathrm{L}$ of methanol with internal standards ( $20 \mu \mathrm{mol}$ of both methionine sulfur and camphor 10-sulfonic acid). Solutions were mixed and $400 \mu \mathrm{L}$ of Milli-Q water (Millipore, Billerica, MA, USA) and $1 \mathrm{~mL}$ of chloroform was added, followed by centrifugation at $4600 \times g$ for $5 \mathrm{~min}$ at $4{ }^{\circ} \mathrm{C}$. Next, the aqueous layer was transferred to a 5-kDa cutoff centrifugal filter tube (Millipore, Billerica, MA, USA) to remove large molecules. The filtrate was then centrifugally concentrated at $35^{\circ} \mathrm{C}$ and reconstituted with $50 \mu \mathrm{L}$ of Milli-Q water that contained reference compounds $(200 \mu \mathrm{moL} / \mathrm{L}$ each of 3-aminopyrrolidine and trimesic acid) prior to CE-TOFMS.

\subsubsection{Capillary Electrophoresis Time-of-Flight Mass Spectrometry (CE-TOFMS)}

Analysis using CE-TOFMS to quantify cationic and anionic metabolites has been described previously [82-84]. Briefly, CE-TOFMS analysis was used an Agilent CE capillary electrophoresis system equipped with an Agilent 6210 time-of-flight mass spectrometer, Agilent 1100 series isocratic HPLC pump, Agilent G1603A CE-MS adapter kit, and Agilent G1607A CE-ESI-MS sprayer kit (Agilent Technologies, Waldbronn, Germany). Agilent G2201AA ChemStation software version B.03.01 for CE 
(Agilent Technologies, Waldbronn, Germany) was used to control systems. Systems were connected by a fused silica capillary ( $50 \mu \mathrm{m}$ internal diameter $\times 100 \mathrm{~cm}$ total length) with $1 \mathrm{~mol} / \mathrm{L}$ formic acid as the electrolyte for cationic analysis and $50 \mathrm{mmol} / \mathrm{L}$ ammonium acetate solution for anionic analysis. The spectrometer was scanned from $m / z 50$ to 1000 [85]. MasterHands automatic integration software (Keio University, Tsuruoka, Yamagata, Japan) was used to extract peaks in order to quantify $\mathrm{m} / \mathrm{z}$, peak area, and migration time (MT) [84]. Signal peaks corresponding to isotopomers, adduct ions, and other product ions of known metabolites were excluded. The remaining peaks were annotated by matching $\mathrm{m} / \mathrm{z}$ values and normalized migration times of corresponding authentic standard compounds [81].

As described previously [86], CE-TOFMS analysis enabled measurement of the absolute concentrations of metabolites on the basis of their peak area and a 6-point calibration curve for each metabolite. Quantification was performed using the $(\mathrm{M}+\mathrm{H})^{+}$or $(\mathrm{M}-\mathrm{H})^{-}$parent ion peak area for each metabolite compared to the same parent peak in the standard solution. Concentration is only reported if the measured area is above the signal:noise ratio $(\mathrm{S} / \mathrm{N})$ of 5 and peak area is within linear range of the standard curve [87]. As reported previously [88], coefficients of variation (CVs) for repeat technical was less than $10 \%$. In this study, 103 distinct metabolites were originally detected by CE-TOFMS. Metabolite concentrations are reported in $\mu \mathrm{M}$.

\subsection{Metabolite Classifications}

Among the 82 metabolites included in analyses, we identified metabolite classes and primary pathways using a three-step process (summarized in Figure 3). We first categorized metabolites based on the Human Metabolome Database (HMDB) designation of metabolite class, subclass, and direct parent [89]. Table S1 includes classifications and groupings of all metabolites. The "class" classification is included in the tables in the Results section for significant metabolites.

Second, after identifying the 18 overlapping metabolites associated with MetS in both cohorts, we used the HMDB pathway database to identify the biochemical pathways each individual metabolite participates in as well as the pathways that were overlapping across metabolites. Third, we conducted a comprehensive literature search to identify which of the overlapping pathways were associated with MetS and/or related conditions. The primary pathway for metabolites highlighted in the tables in the Results section for significant metabolites included the pathways identified by HMDB with primary biologic relevance to MetS.

\subsection{Outcomes}

\subsubsection{Definition of MetS}

Metabolic syndrome (MetS) was defined using the Third Adults Treatment Panel of the National Cholesterol Education Program (NCEPATPIII) criteria, revised by the American Heart Association and National Heart, Lung, and Blood Institute (AHA/NHLBI) [5]. A diagnosis of MetS required meeting at least three of five criteria/risk factors, including elevated waist circumference, elevated fasting plasma glucose, elevated serum triglyceride levels, reduced serum HDL cholesterol, and elevated blood pressure. Elevated waist circumference was defined as greater than 40 inches in males and greater than 35 inches in females. For TMCS participants, the criterion was modified as appropriate for a Japanese population, defining elevated waist circumference as greater than or equal to $100 \mathrm{~cm}$ in males and greater than or equal to $90 \mathrm{~cm}$ in females [40]. Elevated fasting glucose was defined as greater than or equal to $100 \mathrm{mg} / \mathrm{dL}$ or the use of prescription diabetes medications. Elevated triglyceride level was defined as greater than or equal to $150 \mathrm{mg} / \mathrm{Dl}$ or the use of prescription hyperlipidemia medications. Reduced HDL cholesterol was defined as less than $40 \mathrm{mg} / \mathrm{dl}$ in males, less than $50 \mathrm{mg} / \mathrm{dL}$ in females, or the use of prescription hyperlipidemia medications. Elevated blood pressure was defined as greater than or equal to $130 \mathrm{~mm} \mathrm{Hg}$ systolic blood pressure, greater than or equal to $85 \mathrm{~mm} \mathrm{Hg}$ diastolic blood pressure, or the use of prescription hypertension medications. All prescription medication use in both cohorts were self-reported through a standardized medication questionnaire. 


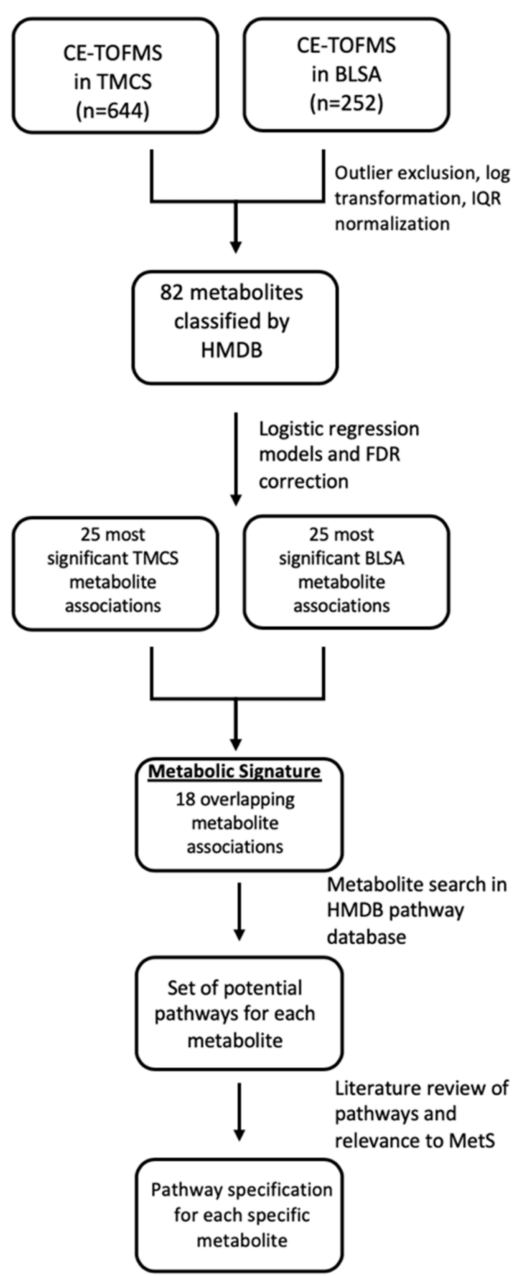

Figure 3. Establishing a metabolic signature of MetS: This figure presents an overview of the methodology, demonstrating data processing steps, analytic methods, and data interpretation including class and pathway classification of metabolites. CE-TOFMS: capillary electrophoresis time-of-flight mass spectrometry; TMCS: Tsuruoka Metabolomics Cohort Study; BLSA: Baltimore Longitudinal Study of Aging; HMDB: Human Metabolome Database; FDR: false-discovery rate.

\subsubsection{Individual Risk Factors}

In the BLSA sample, plasma levels of triglycerides and fasting plasma glucose were measured using previously detailed enzymatic methods $[90,91]$. HDL-C values were measured using a dextran sulfate-magnesium precipitation procedure. In addition to outcomes measured in blood serum, waist circumference and blood pressure in both cohorts were also measured. BLSA staff clinicians measured waist circumference with a tape measure kept parallel to the floor from the hipbone and wrapping around the waist at the level of the umbilicus while participants held their breath [92]. Systolic and diastolic blood pressures were recorded three times in both arms in a seated position using a mercury sphygmomanometer sized to the arm of each participant, and the mean of the systolic and diastolic measurements were used in analysis [93].

In the TMCS sample, serum levels of triglycerides and fasting plasma glucose were measured using enzymatic and hexokinase methods, respectively [94]. HDL-C values were obtained using a direct method. Waist circumference was measured to the nearest $0.1 \mathrm{~cm}$ at the umbilicus at the end of a normal breath. If the umbilicus drooped down, the measurement was recorded midway between the inferior margin of the last rib and the top of the iliac crest in a horizontal plane [40]. Systolic and diastolic blood pressures were each measured twice on one occasion while seated using an automated 
sphygmomanometer (Omron HBP-T105S-N, Kyoto, Kyoto, Japan), and the mean of each of the two measurements were used in analysis [40].

\subsection{Statistical Analyses}

We first excluded all metabolites with $>30 \%$ missing values (i.e., values identified as "not detectable" (ND) due to values lower than the limit of detection (LOD)) [57] and only included metabolites that overlapped between samples; 21 of 103 metabolites were excluded, resulting in 82 total metabolites included in analyses (see Figure 3). Similar to prior work, we then imputed all missing values as the lowest detectable value/2 [95]. In the BLSA cohort, the number of imputed values for individual metabolites ranged from $0(0.0 \%$ of total) to 54 ( $21.4 \%$ of total), with a median of $5(2.0 \%$ of total). In the TMCS cohort, the number of imputed values ranged from $0(0.0 \%$ total $)$ to $174(27.0 \%$ of total), with a median of 12 (1.9\% of total). Following imputation, we compared metabolite values between BLSA and TMCS using nonparametric Wilcoxon rank-sum tests. To account for multiple comparisons, we corrected levels of significance using false discovery rate (FDR)-adjusted $p$-values [96]. We compared demographic characteristics in addition to the prevalence of MetS and its individual risk factors between BLSA and TMCS cohorts using two-sample t-tests for continuous variables and chi-squared tests for categorical variables.

For primary analyses, we first natural log transformed all 82 metabolites and then excluded outlier values outside the 1.5 interquartile range (i.e., $1.5 \times \mathrm{IQR}$ ). We then performed IQR normalization of metabolite values by subtracting the median value from the individual metabolite value and by dividing by the IQR. We used these normalized values in multivariate logistic regression models to explore the associations between distinct blood metabolites (predictors) and the binary outcome indicators of MetS (i.e., present/absent) and its 5 individual risk factors: elevated waist circumference, elevated fasting glucose, elevated triglycerides, reduced HDL cholesterol, and elevated blood pressure. The logistic regression models indicated whether each of the 82 metabolites was associated with either increased or decreased odds of the outcome. Covariates included sex and age at blood draw. For BLSA models, we included storage time as an additional covariate. Model results indicate an increase or decrease in the odds of having MetS or one of its individual risk factors associated with a one-unit increase in the normalized metabolite concentration(s). We corrected levels of significance using FDR-adjusted $p$-values using the same method described previously.

In order to identify a metabolite signature of MetS and to then describe the biologic pathways that may link the metabolites to underlying disease processes, we first ranked metabolites within each cohort. The ranking was based on the likelihood of a type I error (e.g., $p$-value) generated from the logistic regression model, indicating the strength of the association between the metabolite and MetS. The highest-ranking metabolite had the least likelihood of a type I error (i.e., smallest FDR-corrected $p$-value), the second-highest-ranking metabolite had the second smallest $p$-value, and so on. We used this ranking to identify the top 25 metabolites in each cohort. We then visualized the 25 top ranked metabolites for each cohort using side-by-side rainplots [97], which visualize the significance and direction of the odds ratio estimate of each metabolite association. Of these 25 metabolites, we identified which metabolites were shared between cohorts (i.e., in the top 25 for both BLSA and TMCS), which suggest metabolites and associated metabolic pathways that may be intrinsic to MetS despite differences in population-specific factors. We additionally identified metabolites that were unique to a cohort (i.e., in the top 25 for one cohort but not in the other). We included both the a priori indicated HMDB designated "class" classification as well as the primary metabolic "pathway" classification in order to meaningfully group metabolites and to develop a model linking metabolite associations to biological processes.

We performed sensitivity analyses for the multivariate logistic regression models including smoking status, physical activity, and diet (for TMCS only) as covariates in addition to age, sex, and serum sample storage time (for BLSA only). Smoking status was coded 0 (never smoker) or 1 (current or former smoker). Diet quality was assessed using the Dietary Approaches to Stop 
Hypertension (DASH) score [98]. The DASH score indicates adherence to the DASH dietary pattern by measuring consumption of 9 target nutrients including total fat, saturated fat, protein, fiber, cholesterol, calcium, potassium, and magnesium. We excluded magnesium as this target was not included in TMCS data collection. The DASH score therefore represented the sum of 8 nutrient components, with higher values indicating a higher quality diet. The DASH score was not collected for the majority of BLSA participants included in this study and was therefore only included in sensitivity analyses for the TMCS cohort.

Physical activity measurements were obtained via questionnaire in each study. For BLSA participants, a physical activity (PA) questionnaire was administered that asked participants to estimate the time spent performing 97 different activities. The use of this questionnaire in the BLSA has previously been described [99]. PA intensity was measured in metabolic equivalents (METs) [100]. As in prior studies, activities were grouped into low-intensity ( $<4 \mathrm{METs})$, moderate-intensity (4-5.9 METs), and high-intensity ( $>6$ METs) [101]. Total PA was determined by summing the three categories of activity and by multiplying the hours spent in each activity by the assigned MET value. The PA survey administered to TMCS participants was developed initially for the Japan Public Health Center-based Study [102] and relies on measurement of the domains of occupational activity, leisure time activity, sleeping, and other activities [103]. Total PA was determined by summing all domains of activity and by multiplying the hours spent in each activity by the assigned MET value. In both BLSA and TMCS, we used total PA in METs/week in sensitivity analyses.

We conducted additional sensitivity analyses exploring potential sex-specific differences in metabolite associations. We ran sex-stratified multivariate logistic regression models for the BLSA and TMCS including age as a covariate as well as sample storage time in the BLSA cohort. Finally, we conducted sensitivity analyses to verify that imputation did not significantly impact results. We ran multivariate logistic regression models for BLSA and TMCS including sex and age as covariates as well as sample storage time in the BLSA cohort while excluding all imputed data from the analyses.

For all logistic regression models, we reported odds ratios. The type I error level was set to 0.05 for unadjusted $p$-values, and we corrected for multiple comparisons using FDR-adjusted $p$-values as described above. We used R Studio 1.1.453 for all data analyses and visualizations.

Supplementary Materials: The following are available online at http:/www.mdpi.com/1422-0067/21/4/1249/s1, Figure S1: title, Table S1: title, Video S1: title.

Author Contributions: Conceptualization, J.A.R., V.R.V, Y.A., A.O., and M.T.; data curation, V.R.V and P.E.; formal analysis, J.A.R. and C.-W.H..; funding acquisition, M.T.; investigation, T.T. (Toru Takebayashi ), S.H., and M.I.; methodology, J.A.R, V.R.V, Y.A., T.T. (Toshiko Tanaka), T.T. (Toru Takebayashi ), S.H., and M.I.; project administration, L.F. and M.T.; resources, T.T. (Toru Takebayashi ), S.H., M.I., and M.T.; supervision, V.R.V and M.T.; validation, C.-W.H.; visualization, J.A.R.; writing-original draft, J.A.R.; writing-review and editing, V.R.V., A.O., and M.T. All authors have read and agreed to the published version of the manuscript

Funding: This research was supported in part by the Intramural Research Program of the NIH, National Institute on Aging. The funders had no role in study design, data collection and analysis, decision to publish, or preparation of the manuscript. M.T. is grateful for funding support from the Andrew and Lillian A. Posey Foundation to the Clinical and Translational Neuroscience Section, Laboratory of Behavioral Neuroscience, NIA.

Acknowledgments: This study was funded in part by the National Institute on Aging Intramural Research Program. We are grateful to the Baltimore Longitudinal Study of Aging and Tsuruoka Metabolomics Cohort Study participants for their invaluable contributions.

Conflicts of Interest: The authors declare no conflict of interest.

\section{Abbreviations}

BLSA Baltimore Longitudinal Study of Aging

TMCS Tsuruoka Metabolomics Cohort Study

MetS Metabolic Syndrome

CE-TOFMS Capillary electrophoresis time-of-flight mass spectrometry

AD Alzheimer's disease 


$\begin{array}{ll}\text { HMDB } & \text { Human Metabolome Database } \\ \text { FDR } & \text { False-discovery rate } \\ \text { PA } & \text { Physical activity } \\ \text { MET } & \text { Metabolic equivalent } \\ \text { DASH } & \text { Dietary Approaches to Stop Hypertension } \\ \text { LOD } & \text { Limit of detection } \\ \text { IQR } & \text { Interquartile range } \\ \text { BCAA } & \text { Branched-chain amino acid } \\ \text { BCKA } & \text { Branched-chain keto acid } \\ \text { GSH } & \text { Glutathione } \\ \text { TCA } & \text { Tricarboxylic acid }\end{array}$

\section{References}

1. Ford, E.S. Risks for all-cause mortality, cardiovascular disease, and diabetes associated with the metabolic syndrome-A summary of the evidence. Diabetes Care 2005, 28, 1769-1778. [CrossRef] [PubMed]

2. Galassi, A.; Reynolds, K.; He, J. Metabolic syndrome and risk of cardiovascular disease: A meta-analysis. Am. J. Med. 2006, 119, 812-819. [CrossRef] [PubMed]

3. Chen, W.; Pan, Y.; Jing, J.; Zhao, X.; Liu, L.; Meng, X.; Wang, Y.; Wang, Y.; Investigators, C. Recurrent Stroke in Minor Ischemic Stroke or Transient Ischemic Attack With Metabolic Syndrome and/or Diabetes Mellitus. J. Am. Heart Assoc. 2017, 6. [CrossRef] [PubMed]

4. Razay, G.; Vreugdenhil, A.; Wilcock, G. The metabolic syndrome and Alzheimer disease. Arch. Neurol. 2007, 64, 93-96. [CrossRef] [PubMed]

5. Grundy, S.M.; Cleeman, J.I.; Daniels, S.R.; Donato, K.A.; Eckel, R.H.; Franklin, B.A.; Gordon, D.J.; Krauss, R.M.; Savage, P.J.; Smith, S.C., Jr.; et al. Diagnosis and management of the metabolic syndrome: An American Heart Association/National Heart, Lung, and Blood Institute scientific statement: Executive Summary. Crit. Pathw. Cardiol. 2005, 4, 198-203. [CrossRef] [PubMed]

6. Shin, D.; Kongpakpaisarn, K.; Bohra, C. Trends in the prevalence of metabolic syndrome and its components in the United States 2007-2014. Int. J. Cardiol. 2018, 259, 216-219. [CrossRef] [PubMed]

7. Hu, F.B. Globalization of Diabetes The role of diet, lifestyle, and genes. Diabetes Care 2011, 34, $1249-1257$. [CrossRef]

8. Odegaard, A.O.; Koh, W.P.; Yuan, J.M.; Gross, M.D.; Pereira, M.A. Western-Style Fast Food Intake and Cardiometabolic Risk in an Eastern Country. Circulation 2012, 126, 182-188. [CrossRef]

9. Ministry of Health LaWoJ. A Summary of the National Nutritional and Health Survey of 2016. Available online: https://www.mhlw.go.jp/bunya/kenkou/eiyou/dl/h28-houkoku.pdf (accessed on 10 November 2019).

10. Scholze, J.; Alegria, E.; Ferri, C.; Langham, S.; Stevens, W.; Jeffries, D.; Uhl-Hochgraeber, K. Epidemiological and economic burden of metabolic syndrome and its consequences in patients with hypertension in Germany, Spain and Italy; a prevalence-based model. BMC Public Health 2010, 10. [CrossRef]

11. Dekker, J.M.; Girman, C.; Rhodes, T.; Nijpels, G.; Stehouwer, C.D.A.; Bouter, L.M.; Heine, R.J. Metabolic syndrome and 10-year cardiovascular disease risk in the hoorn study. Circulation 2005, 112, 666-673. [CrossRef]

12. Exalto, L.G.; van der Flier, W.M.; van Boheemen, C.J.M.; Kappelle, L.J.; Vrenken, H.; Teunissen, C.; Koene, T.; Scheltens, P.; Biessels, G.J. The metabolic syndrome in a memory clinic population: Relation with clinical profile and prognosis. J. Neurol. Sci. 2015, 351, 18-23. [CrossRef] [PubMed]

13. Raffaitin, C.; Gin, H.; Empana, J.P.; Helmer, C.; Berr, C.; Tzourio, C.; Portet, F.; Dartigues, J.F.; Alperovitch, A.; Barberger-Gateau, P. Metabolic Syndrome and Risk for Incident Alzheimer's Disease or Vascular Dementia The Three-City Study. Diabetes Care 2009, 32, 169-174. [CrossRef] [PubMed]

14. Vanhanen, M.; Koivisto, K.; Moilanen, L.; Helkala, E.L.; Hanninen, T.; Soininen, H.; Kervinen, K.; Kesaniemi, A.; Laakso, M.; Kuusisto, J. Association of metabolic syndrome with Alzheimer disease A population-based study. Neurology 2006, 67, 843-847. [CrossRef]

15. Luque-Contreras, D.; Carvajal, K.; Toral-Rios, D.; Franco-Bocanegra, D.; Campos-Pena, V. Oxidative Stress and Metabolic Syndrome: Cause or Consequence of Alzheimer's Disease? Oxid. Med. Cell. Longev. 2014. [CrossRef] [PubMed] 
16. Hasin, Y.; Seldin, M.; Lusis, A. Multi-omics approaches to disease. Genome Biol. 2017, 18, 83. [CrossRef]

17. Johnson, C.H.; Ivanisevic, J.; Siuzdak, G. Metabolomics: Beyond biomarkers and towards mechanisms. Nat. Rev. Mol. Cell Biol. 2016, 17, 451-459. [CrossRef]

18. Sallese, A.; Zhu, J.J. Mass spectrometry based metabolomics: A novel analytical technique for detecting metabolic syndrome? Bioanalysis 2017, 9, 1623-1626. [CrossRef]

19. Pujos-Guillot, E.; Brandolini, M.; Petera, M.; Grissa, D.; Joly, C.; Lyan, B.; Herquelot, E.; Czernichow, S.; Zins, M.; Goldberg, M.; et al. Systems Metabolomics for Prediction of Metabolic Syndrome. J. Proteome Res. 2017, 16, 2262-2272. [CrossRef]

20. Wiklund, P.K.; Pekkala, S.; Autio, R.; Munukka, E.; Xu, L.T.; Saltevo, J.; Cheng, S.M.; Kujala, U.M.; Alen, M.; Cheng, S.L. Serum metabolic profiles in overweight and obese women with and without metabolic syndrome. Diabetol. Metab. Syndr. 2014, 6. [CrossRef]

21. Zhong, F.Y.; Xu, M.Y.; Bruno, R.S.; Ballard, K.D.; Zhu, J.J. Targeted High Performance Liquid Chromatography Tandem Mass Spectrometry-based Metabolomics differentiates metabolic syndrome from obesity. Exp. Biol. Med. 2017, 242, 773-780. [CrossRef]

22. Tulipani, S.; Llorach, R.; Jauregui, O.; Lopez-Uriarte, P.; Garcia-Aloy, M.; Bullo, M.; Salas-Salvado, J.; Andres-Lacueva, C. Metabolomics Unveils Urinary Changes in Subjects with Metabolic Syndrome following 12-Week Nut Consumption. J. Proteome Res. 2011, 10, 5047-5058. [CrossRef] [PubMed]

23. Pallister, T.; Jackson, M.A.; Martin, T.C.; Zierer, J.; Jennings, A.; Mohney, R.P.; MacGregor, A.; Steves, C.J.; Cassidy, A.; Spector, T.D.; et al. Hippurate as a metabolomic marker of gut microbiome diversity: Modulation by diet and relationship to metabolic syndrome. Sci. Rep. 2017, 7. [CrossRef] [PubMed]

24. Lent-Schochet, D.; McLaughlin, M.; Ramakrishnan, N.; Jialal, I. Exploratory metabolomics of metabolic syndrome: A status report. World J. Diabetes 2019, 10, 23-36. [CrossRef] [PubMed]

25. Lee, C.M.Y.; Huxley, R.R.; Woodward, M.; Zimmet, P.; Shaw, J.; Cho, N.H.; Kim, H.R.; Viali, S.; Tominaga, M.; Vistisen, D.; et al. The metabolic syndrome identifies a heterogeneous group of metabolic component combinations in the Asia-Pacific region. Diabetes Res. Clin. Pr. 2008, 81, 377-380. [CrossRef] [PubMed]

26. Van Valkengoed, I.G.M.; Argmann, C.; Ghauharali-van der Vlugt, K.; Aerts, J.M.F.G.; Brewster, L.M.; Peters, R.J.G.; Vaz, F.M.; Houtkooper, R.H. Ethnic differences in metabolite signatures and type 2 diabetes: A nested case-control analysis among people of South Asian, African and European origin. Nutr. Diabetes 2017, 7. [CrossRef] [PubMed]

27. Tillin, T.; Hughes, A.D.; Wang, Q.; Wurtz, P.; Ala-Korpela, M.; Sattar, N.; Forouhi, N.G.; Godsland, I.F.; Eastwood, S.V.; McKeigue, P.M.; et al. Diabetes risk and amino acid profiles: Cross-sectional and prospective analyses of ethnicity, amino acids and diabetes in a South Asian and European cohort from the SABRE (Southall And Brent REvisited) Study. Diabetologia 2015, 58, 968-979. [CrossRef] [PubMed]

28. Teramoto, T.; Uno, K.; Miyoshi, I.; Khan, I.; Gorcyca, K.; Sanchez, R.J.; Yoshida, S.; Mawatari, K.; Masaki, T.; Arai, H.; et al. Low-density lipoprotein cholesterol levels and lipid-modifying therapy prescription patterns in the real world: An analysis of more than 33,000 high cardiovascular risk patients in Japan. Atherosclerosis 2016, 251, 248-254. [CrossRef]

29. Hyman, D.J.; Pavlik, V.N. Self-reported hypertension treatment practices among primary care physicians-Blood pressure thresholds, drug choices, and the role of guidelines and evidence-based medicine. Arch. Intern. Med. 2000, 160, 2281-2286. [CrossRef]

30. Zhou, B.; Danaei, G.; Stevens, G.A.; Bixby, H.; Taddei, C.; Carrillo-Larco, R.M.; Solomon, B.; Riley, L.M.; Di Cesare, M.; Iurilli, M.L.C.; et al. Long-term and recent trends in hypertension awareness, treatment, and control in 12 high-income countries: An analysis of 123 nationally representative surveys. Lancet 2019, 394, 639-651. [CrossRef]

31. Islami, F.; Torre, L.A.; Jemal, A. Global trends of lung cancer mortality and smoking prevalence. Transl. Lung Cancer $R$ 2015, 4, 327-338. [CrossRef]

32. Newgard, C.B.; An, J.; Bain, J.R.; Muehlbauer, M.J.; Stevens, R.D.; Lien, L.F.; Haqq, A.M.; Shah, S.H.; Arlotto, M.; Slentz, C.A.; et al. A Branched-Chain Amino Acid-Related Metabolic Signature that Differentiates Obese and Lean Humans and Contributes to Insulin Resistance. Cell Metab. 2009, 9, 565-566. [CrossRef]

33. Flores-Guerrero, J.L.; Oste, M.C.J.; Kieneker, L.M.; Gruppen, E.G.; Wolak-Dinsmore, J.; Otvos, J.D.; Connelly, M.A.; Bakker, S.J.L.; Dullaart, R.P.F. Plasma Branched-Chain Amino Acids and Risk of Incident Type 2 Diabetes: Results from the PREVEND Prospective Cohort Study. J. Clin. Med. 2018, 7. [CrossRef] [PubMed] 
34. Patel, M.J.; Batch, B.C.; Svetkey, L.P.; Bain, J.R.; Turer, C.B.; Haynes, C.; Muehlbauer, M.J.; Stevens, R.D.; Newgard, C.B.; Shah, S.H. Race and Sex Differences in Small-Molecule Metabolites and Metabolic Hormones in Overweight and Obese Adults. Omics 2013, 17, 627-635. [CrossRef] [PubMed]

35. Sun, L.; Hu, C.Y.; Yang, R.Y.; Lv, Y.; Yuan, H.P.; Liang, Q.H.; He, B.J.; Pang, G.F.; Jiang, M.H.; Dong, J.; et al. Association of circulating branched-chain amino acids with cardiometabolic traits differs between adults and the oldest-old. Oncotarget 2017, 8, 88882-88893. [CrossRef]

36. Lotta, L.A.; Scott, R.A.; Sharp, S.J.; Burgess, S.; Luan, J.A.; Tillin, T.; Schmidt, A.F.; Imamura, F.; Stewart, I.D.; Perry, J.R.B.; et al. Genetic Predisposition to an Impaired Metabolism of the Branched-Chain Amino Acids and Risk of Type 2 Diabetes: A Mendelian Randomisation Analysis. PLoS Med. 2016, 13. [CrossRef]

37. Jackson, R.H.; Singer, T.P. Inactivation of the 2-Ketoglutarate and Pyruvate-Dehydrogenase Complexes of Beef-Heart by Branched-Chain Keto Acids. J. Biol. Chem. 1983, 258, 1857-1865.

38. Walajtysrode, E.; Williamson, J.R. Effects of Branched-Chain Alpha-Ketoacids on the Metabolism of Isolated Rat-Liver Cells. 3. Interactions with Pyruvate-Dehydrogenase. J. Biol. Chem. 1980, 255, 413-418.

39. Oyarzabal, A.; Martinez-Pardo, M.; Merinero, B.; Navarrete, R.; Desviat, L.R.; Ugarte, M.; Rodriguez-Pombo, P. A Novel Regulatory Defect in the Branched-Chain -Keto Acid Dehydrogenase Complex Due to a Mutation in the PPM1K Gene Causes a Mild Variant Phenotype of Maple Syrup Urine Disease. Hum. Mutat. 2013, 34, 355-362. [CrossRef]

40. Iida, M.; Harada, S.; Kurihara, A.; Fukai, K.; Kuwabara, K.; Sugiyama, D.; Takeuchi, A.; Okamura, T.; Akiyama, M.; Nishiwaki, Y.; et al. Profiling of plasma metabolites in postmenopausal women with metabolic syndrome. Menopause 2016, 23, 749-758. [CrossRef]

41. Lustgarten, M.S.; Price, L.L.; Chale, A.; Phillips, E.M.; Fielding, R.A. Branched Chain Amino Acids Are Associated With Muscle Mass in Functionally Limited Older Adults. J. Gerontol. A Biol. 2014, 69, 717-724. [CrossRef]

42. Qin, L.Q.; Xun, P.C.; Bujnowski, D.; Daviglus, M.L.; Van Horn, L.; Stamler, J.; He, K.; Grp, I.C.R. Higher Branched-Chain Amino Acid Intake Is Associated with a Lower Prevalence of Being Overweight or Obese in Middle-Aged East Asian and Western Adults. J. Nutr. 2011, 141, 249-254. [CrossRef] [PubMed]

43. Wang, J.; Liu, Y.X.; Lian, K.; Shentu, X.Y.; Fang, J.W.; Shao, J.; Chen, M.P.; Wang, Y.B.; Zhou, M.Y.; Sun, H.P. BCAA Catabolic Defect Alters Glucose Metabolism in Lean Mice. Front. Physiol. 2019, 10. [CrossRef] [PubMed]

44. Tamanna, N.; Mahmood, N. Emerging Roles of Branched-Chain Amino Acid Supplementation in Human Diseases. Int. Sch. Res. Not. 2014, 2014, 235619. [CrossRef] [PubMed]

45. Wang, F.H.; Liu, J.; Deng, Q.J.; Qi, Y.; Wang, M.; Wang, Y.; Zhang, X.G.; Zhao, D. Association between plasma essential amino acids and atherogenic lipid profile in a Chinese population: A cross-sectional study. Atherosclerosis 2019, 286, 7-13. [CrossRef] [PubMed]

46. Solon-Biet, S.M.; Cogger, V.C.; Pulpitel, T.; Wahl, D.; Clark, X.; Bagley, E.; Gregoriou, G.C.; Senior, A.M.; Wang, Q.P.; Brandon, A.E.; et al. Branched chain amino acids impact health and lifespan indirectly via amino acid balance and appetite control. Nat. Metab. 2019, 1, 532-545. [CrossRef]

47. Funchal, C.; Rosa, A.M.; Wajner, M.; Wofchuk, S.; Pureur, R.P. Reduction of glutamate uptake into cerebral cortex of developing rats by the branched-chain alpha-keto acids accumulating in maple syrup urine disease. Neurochem. Res. 2004, 29, 747-753. [CrossRef]

48. Scott, H.A.; Gebhardt, F.M.; Mitrovic, A.D.; Vandenberg, R.J.; Dodd, P.R. Glutamate transporter variants reduce glutamate uptake in Alzheimer's disease. Neurobiol. Aging 2011, 32. [CrossRef]

49. Li, H.J.; Ye, D.; Xie, W.; Hua, F.; Yang, Y.L.; Wu, J.; Gu, A.F.; Ren, Y.; Mao, K.S. Defect of branched-chain amino acid metabolism promotes the development of Alzheimer's disease by targeting the mTOR signaling. Biosci. Rep. 2018, 38. [CrossRef]

50. Larsson, S.C.; Markus, H.S. Branched-chain amino acids and Alzheimer's disease: A Mendelian randomization analysis. Sci. Rep. 2017, 7. [CrossRef]

51. Toledo,J.B.; Arnold, M.; Kastenmuller, G.; Chang, R.; Baillie, R.A.; Han, X.L.; Thambisetty, M.; Tenenbaum, J.D.; Suhre, K.; Thompson, J.W.; et al. Metabolic network failures in Alzheimer's disease: A biochemical road map. Alzheimers Dement. 2017, 13, 965-984. [CrossRef]

52. Lovell, M.A.; Markesbery, W.R. Oxidative DNA damage in mild cognitive impairment and late-stage Alzheimers disease. Nucleic Acids Res. 2007, 35, 7497-7504. [CrossRef] 
53. Nunomura, A.; Castellani, R.J.; Zhu, X.W.; Moreira, P.I.; Perry, G.; Smith, M.A. Involvement of oxidative stress in Alzheimer disease. J. Neuropathol. Exp. Neurol. 2006, 65, 631-641. [CrossRef] [PubMed]

54. Furukawa, S.; Fujita, T.; Shimabukuro, M.; Iwaki, M.; Yamada, Y.; Nakajima, Y.; Nakayama, O.; Makishima, M.; Matsuda, M.; Shimomura, I. Increased oxidative stress in obesity and its impact on metabolic syndrome. J. Clin. Investig. 2004, 114, 1752-1761. [CrossRef]

55. Sastre, J.; Pallardo, F.V.; Llopis, J.; Furukawa, T.; Vina, J.R.; Vina, J. Glutathione Depletion by Hyperphagia-Induced Obesity. Life Sci. 1989, 45, 183-187. [CrossRef]

56. Bains, J.S.; Shaw, C.A. Neurodegenerative disorders in humans: The role of glutathione in oxidative stress-mediated neuronal death. Brain Res. Rev. 1997, 25, 335-358. [CrossRef]

57. Mahajan, U.V.; Varma, V.R.; Griswold, M.E.; Blackshear, C.T.; An, Y.; Oommen, A.M.; Varma, S.; Troncoso, J.C.; Pletnikova, O.; O’Brien, R.; et al. Dysregulation of multiple metabolic networks related to brain transmethylation and polyamine pathways in Alzheimer's disease: A targeted metabolomic and transcriptomic study. PLoS Med. 2020, 17, e1003012. [CrossRef]

58. Wu, G.Y.; Fang, Y.Z.; Yang, S.; Lupton, J.R.; Turner, N.D. Glutathione metabolism and its implications for health. J. Nutr. 2004, 134, 489-492. [CrossRef]

59. Elshorbagy, A.K.; Valdivia-Garcia, M.; Refsum, H.; Butte, N. The Association of Cysteine with Obesity, Inflammatory Cytokines and Insulin Resistance in Hispanic Children and Adolescents. PLoS ONE 2012, 7. [CrossRef]

60. Lord, R.S.; Bralley, J.A. Clinical Applications of Urinary Organic Acids. Part 1: Detoxification Markers. Altern Med. Rev. 2008, 13, 205-215.

61. Ruiz-Ramirez, A.; Ortiz-Balderas, E.; Gardozo-Saldana, G.; Diaz-Diaz, E.; El-Hafidi, M. Glycine restores glutathione and protects against oxidative stress in vascular tissue from sucrose-fed rats. Clin. Sci. 2014, 126, 19-29. [CrossRef]

62. Zhou, X.H.; He, L.Q.; Wu, C.R.; Zhang, Y.M.; Wu, X.; Yin, Y.L. Serine alleviates oxidative stress via supporting glutathione synthesis and methionine cycle in mice. Mol. Nutr. Food Res. 2017, 61, 1700262. [CrossRef] [PubMed]

63. Sappington, D.; Penney, R.; Siegel, E.; Boysen, G. Glutamine drives glutathione synthesis and contributes to radiation sensitivity of A549 and H460 lung cancer cell lines. Cancer Res. 2016, 76, 1041. [CrossRef] [PubMed]

64. Felig, P. The glucose-alanine cycle. Metabolism 1973, 22, 179-207. [CrossRef]

65. Jansson, P.A.; Larsson, A.; Smith, U.; Lonnroth, P. Lactate Release from the Subcutaneous Tissue in Lean and Obese Men. J. Clin. Investig. 1994, 93, 240-246. [CrossRef]

66. Yang, C.D.; Ko, B.; Hensley, C.T.; Jiang, L.; Wasti, A.T.; Kim, J.; Sudderth, J.; Calvaruso, M.A.; Lumata, L.; Mitsche, M.; et al. Glutamine Oxidation Maintains the TCA Cycle and Cell Survival during Impaired Mitochondrial Pyruvate Transport. Mol. Cell 2014, 56, 414-424. [CrossRef]

67. Satapati, S.; Sunny, N.E.; Kucejova, B.; Fu, X.R.; He, T.T.; Mendez-Lucas, A.; Shelton, J.M.; Perales, J.C.; Browning, J.D.; Burgess, S.C. Elevated TCA cycle function in the pathology of diet-induced hepatic insulin resistance and fatty liver. J. Lipid Res. 2012, 53, 1080-1092. [CrossRef]

68. Libert, D.M.; Nowacki, A.S.; Natowicz, M.R. Metabolomic analysis of obesity, metabolic syndrome, and type 2 diabetes: Amino acid and acylcarnitine levels change along a spectrum of metabolic wellness. PeerJ 2018, 6. [CrossRef]

69. Buckley, W.T.; Milligan, L.P. Participation of cysteine and cystine in inactivation of tyrosine aminotransferase in rat liver homogenates. Biochem. J. 1978, 176, 449-454. [CrossRef]

70. Liu, L.L.; Li, Q.; Li, N.J.; Ling, J.H.; Liu, R.; Wang, Y.X.; Sun, L.X.; Chen, X.H.; Bi, K.S. Simultaneous determination of catecholamines and their metabolites related to Alzheimer's disease in human urine. J. Sep. Sci. 2011, 34, 1198-1204. [CrossRef]

71. Fernstrom, J.D.; Fernstrom, M.H. Tyrosine, phenylalanine, and catecholamine synthesis and function in the brain. J. Nutr. 2007, 137, 1539s-1547s. [CrossRef]

72. Gao, X.; Zhang, W.D.; Wang, Y.B.; Pedram, P.; Cahill, F.; Zhai, G.J.; Randell, E.; Gulliver, W.; Sun, G. Serum metabolic biomarkers distinguish metabolically healthy peripherally obese from unhealthy centrally obese individuals. Nutr. Metab. 2016, 13. [CrossRef]

73. Wang, T.J.; Ngo, D.; Psychogios, N.; Dejam, A.; Larson, M.G.; Vasan, R.S.; Ghorbani, A.; O'Sullivan, J.; Cheng, S.; Rhee, E.P.; et al. 2-Aminoadipic acid is a biomarker for diabetes risk. J. Clin. Investig. 2013, 123, 4309-4317. [CrossRef] 
74. Mahajan, U.V.; Varma, V.R.; Huang, C.-W.; An, Y.; Tanaka, T.; Ferrucci, L.; Takebayashi, T.; Harada, S.; Iida, M.; Legido-Quigley, C.; et al. Blood metabolite signatures of metabolic syndrome in two cross-cultural older adult cohorts. 2020. In press.

75. Pasini, E.; Corsetti, G.; Aquilani, R.; Romano, C.; Picca, A.; Calvani, R.; Dioguardi, F.S. Protein-Amino Acid Metabolism Disarrangements: The Hidden Enemy of Chronic Age-Related Conditions. Nutrients 2018, 10. [CrossRef]

76. Ferrucci, L. The Baltimore Longitudinal Study of Aging (BLSA): A 50-year-long journey and plans for the future. J. Gerontol. A Biol. Sci. Med. Sci. 2008, 63, 1416-1419. [CrossRef]

77. Resnick, S.M.; Pham, D.L.; Kraut, M.A.; Zonderman, A.B.; Davatzikos, C. Longitudinal magnetic resonance imaging studies of older adults: A shrinking brain. J. Neurosci. 2003, 23, 3295-3301. [CrossRef]

78. Harada, S.; Takebayashi, T.; Kurihara, A.; Akiyama, M.; Suzuki, A.; Hatakeyama, Y.; Sugiyama, D.; Kuwabara, K.; Takeuchi, A.; Okamura, T.; et al. Metabolomic profiling reveals novel biomarkers of alcohol intake and alcohol-induced liver injury in community-dwelling men. Environ. Health Prev. 2016, 21, 283-284. [CrossRef]

79. Casanova, R.; Varma, S.; Simpson, B.; Kim, M.; An, Y.; Saldana, S.; Riveros, C.; Moscato, P.; Griswold, M.; Sonntag, D.; et al. Blood metabolite markers of preclinical Alzheimer's disease in two longitudinally followed cohorts of older individuals. Alzheimers Dement. 2016, 12, 815-822. [CrossRef]

80. Kuehnbaum, N.L.; Kormendi, A.; Britz-McKibbin, P. Multisegment Injection-Capillary Electrophoresis-Mass Spectrometry: A High-Throughput Platform for Metabolomics with High Data Fidelity. Anal. Chem. 2013, 85, 10664-10669. [CrossRef]

81. Hirayama, A.; Nakashima, E.; Sugimoto, M.; Akiyama, S.; Sato, W.; Maruyama, S.; Matsuo, S.; Tomita, M.; Yuzawa, Y.; Soga, T. Metabolic profiling reveals new serum biomarkers for differentiating diabetic nephropathy. Anal. Bioanal. Chem. 2012, 404, 3101-3109. [CrossRef]

82. Hirayama, A.; Tomita, M.; Soga, T. Sheathless capillary electrophoresis-mass spectrometry with a high-sensitivity porous sprayer for cationic metabolome analysis. Analyst 2012, 137, 5026-5033. [CrossRef]

83. Hirayama, A.; Soga, T. Capillary Electrophoresis-Mass Spectrometry (CE-MS): Principles and Applications; Wiley-VCH: Weinheim, Germany, 2016. [CrossRef]

84. Sugimoto, M.; Wong, D.T.; Hirayama, A.; Soga, T.; Tomita, M. Capillary electrophoresis mass spectrometry-based saliva metabolomics identified oral, breast and pancreatic cancer-specific profiles. Metabolomics 2010, 6, 78-95. [CrossRef]

85. Ohashi, Y.; Hirayama, A.; Ishikawa, T.; Nakamura, S.; Shimizu, K.; Ueno, Y.; Tomita, M.; Soga, T. Depiction of metabolome changes in histidine-starved Escherichia coli by CE-TOFMS. Mol. Biosyst. 2008, 4, 135-147. [CrossRef]

86. Soga, T.; Heiger, D.N. Amino acid analysis by capillary electrophoresis electrospray ionization mass spectrometry. Anal. Chem. 2000, 72, 1236-1241. [CrossRef]

87. Sasaki, K.; Sagawa, H.; Suzuki, M.; Yamamoto, H.; Tomita, M.; Soga, T.; Ohashi, Y. Metabolomics Platform with Capillary Electrophoresis Coupled with High-Resolution Mass Spectrometry for Plasma Analysis. Anal. Chem. 2019, 91, 1295-1301. [CrossRef]

88. Soga, T.; Igarashi, K.; Ito, C.; Mizobuchi, K.; Zimmermann, H.P.; Tomita, M. Metabolomic Profiling of Anionic Metabolites by Capillary Electrophoresis Mass Spectrometry. Anal. Chem. 2009, 81, 6165-6174. [CrossRef]

89. Wishart, D.S.; Feunang, Y.D.; Marcu, A.; Guo, A.C.; Liang, K.; Vazquez-Fresno, R.; Sajed, T.; Johnson, D.; Li, C.R.; Karu, N.; et al. HMDB 4.0: The human metabolome database for 2018. Nucleic Acids Res. 2018, 46, D608-D617. [CrossRef]

90. Metter, E.J.; Windham, B.G.; Maggio, M.; Simonsick, E.M.; Ling, S.M.; Egan, J.M.; Ferrucci, L. Glucose and insulin measurements from the oral glucose tolerance test and mortality prediction. Diabetes Care 2008, 31, 1026-1030. [CrossRef]

91. Mielke, M.M.; Bandaru, V.V.; Han, D.; An, Y.; Resnick, S.M.; Ferrucci, L.; Haughey, N.J. Factors affecting longitudinal trajectories of plasma sphingomyelins: The Baltimore Longitudinal Study of Aging. Aging Cell 2015, 14, 112-121. [CrossRef]

92. Beydoun, M.A.; Tanaka, T.; Beydoun, H.A.; Ding, E.L.; Ferrucci, L.; Zonderman, A.B. Vitamin D receptor and megalin gene polymorphisms are associated with central adiposity status and changes among US adults. J. Nutr. Sci. 2013, 2. [CrossRef] 
93. Brant, L.J.; Ferrucci, L.; Sheng, S.L.; Concin, H.; Zonderman, A.B.; Kelleher, C.C.; Longo, D.L.; Ulmer, H.; Strasak, A.M. Gender Differences in the Accuracy of Time-Dependent Blood Pressure Indices for Predicting Coronary Heart Disease: A Random-Effects Modeling Approach. Gend. Med. 2010, 7, 616-627. [CrossRef] [PubMed]

94. Nakamura, M.; Iso, H.; Kitamura, A.; Imano, H.; Noda, H.; Kiyama, M.; Sato, S.; Yamagishi, K.; Nishimura, K.; Nakai, M.; et al. Comparison between the triglycerides standardization of routine methods used in Japan and the chromotropic acid reference measurement procedure used by the CDC Lipid Standardization Programme. Ann. Clin. Biochem. 2016, 53, 632-639. [CrossRef] [PubMed]

95. Varma, V.R.; Oommen, A.M.; Varma, S.; Casanova, R.; An, Y.; Andrews, R.M.; O’Brien, R.; Pletnikova, O.; Troncoso, J.C.; Toledo, J.; et al. Brain and blood metabolite signatures of pathology and progression in Alzheimer disease: A targeted metabolomics study. PLoS Med. 2018, 15. [CrossRef] [PubMed]

96. Benjamini, Y.; Hochberg, Y. Controlling the False Discovery Rate-A Practical and Powerful Approach to Multiple Testing. J. R. Stat. Soc. B 1995, 57, 289-300. [CrossRef]

97. Henglin, M.; Niiranen, T.; Watrous, J.D.; Lagerborg, K.A.; Antonelli, J.; Claggett, B.L.; Demosthenes, E.J.; Von Jeinsen, B.; Demler, O.; Vasan, R.S.; et al. A Single Visualization Technique for Displaying Multiple Metabolite-Phenotype Associations. Metabolites 2019, 9. [CrossRef]

98. Sacks, F.M.; Appel, L.J.; Moore, T.J.; Obarzanek, E.; Vollmer, W.M.; Svetkey, L.P.; Bray, G.A.; Vogt, T.M.; Cutler, J.A.; Windhauser, M.M.; et al. A dietary approach to prevent hypertension: A review of the Dietary Approaches to Stop Hypertension (DASH) study. Clin. Cardiol. 1999, 22, 6-10. [CrossRef]

99. Verbrugge, L.M.; Gruber-Baldini, A.L.; Fozard, J.L. Age differences and age changes in activities: Baltimore Longitudinal Study of Aging. J. Gerontol. B Psychol. Sci. Soc. Sci. 1996, 51, S30-S41. [CrossRef]

100. Talbot, L.A.; Metter, E.J.; Fleg, J.L. Leisure-time physical activities and their relationship to cardiorespiratory fitness in healthy men and women 18-95 years old. Med. Sci. Sports Exerc. 2000, 32, 417-425. [CrossRef]

101. Talbot, L.A.; Fleg, J.L.; Metter, E.J. Secular trends in leisure-time physical activity in men and women across four decades. Prev. Med. 2003, 37, 52-60. [CrossRef]

102. Baba, S.; Mannami, T.; Konishi, M.; Sasaki, S. Anthropometric measures, blood pressure and major laboratory examination results in the health check-up examination among the JPHC study participants at baseline survey. Japan Public Health Center-based Prospective Study on Cancer and Cardiovascular Diseases. J. Epidemiol. 2001, 11, S87-S93. [CrossRef]

103. Fujii, H.; Yamamoto, S.; Takeda-Imai, F.; Inoue, M.; Tsugane, S.; Kadowaki, T.; Noda, M. Validity and applicability of a simple questionnaire for the estimation of total and domain-specific physical activity. Diabetol. Int. 2011, 2, 47-54. [CrossRef]

(C) 2020 by the authors. Licensee MDPI, Basel, Switzerland. This article is an open access article distributed under the terms and conditions of the Creative Commons Attribution (CC BY) license (http://creativecommons.org/licenses/by/4.0/). 